\title{
Identification of neglected cestode Taenia multiceps microRNAs by illumina sequencing and bioinformatic analysis
}

\author{
Xuhang Wu', Yan Fu', Deying Yang ${ }^{1}$, Yue Xie ${ }^{1}$, Runhui Zhang ${ }^{1}$, Wanpeng Zheng ${ }^{1}$, Huaming Nie ${ }^{1}$, Ning Yan ${ }^{1}$, \\ Ning Wang ${ }^{1}$, Jiahai Wang ${ }^{1}$, Xiaobin $\mathrm{Gu}^{1}$, Shuxian Wang ${ }^{1}$, Xuerong Peng ${ }^{2}$ and Guangyou Yang ${ }^{1 *}$
}

\begin{abstract}
Background: Worldwide, but especially in developing countries, coenurosis of sheep and other livestock is caused by Taenia multiceps larvae, and zoonotic infections occur in humans. Infections frequently lead to host death, resulting in huge socioeconomic losses. MicroRNAs (miRNAs) have important roles in the post-transcriptional regulation of a large number of animal genes by imperfectly binding target mRNAs. To date, there have been no reports of miRNAs in T. multiceps.

Results: In this study, we obtained 12.8 million high quality raw reads from adult T. multiceps small RNA library using Illumina sequencing technology. A total of 796 conserved miRNA families (containing 1,006 miRNAs) from 170,888 unique miRNAs were characterized using miRBase (Release 17.0). Here, we selected three conserved miRNA/miRNA* (antisense strand) duplexes at random and amplified their corresponding precursors using a PCR-based method. Furthermore, 20 candidate novel miRNA precursors were verified by genomic PCR. Among these, six corresponding T. multiceps miRNAs are considered specific for Taeniidae because no homologs were found in other species annotated in miRBase. In addition, 181,077 target sites within T. multiceps transcriptome were predicted for 20 candidate newly miRNAs.
\end{abstract}

Conclusions: Our large-scale investigation of miRNAs in adult T. multiceps provides a substantial platform for improving our understanding of the molecular regulation of T. multiceps and other cestodes development.

Keywords: MicroRNA, Taenia multiceps, Unigene, Illumina sequencing, Candidate novel miRNA

\section{Background}

Increasing evidence shows that control of gene expression is essential for normal development of organisms and for regulating fundamental biological processes [1-4]. Regulation of gene expression occurs at the transcriptional and post-transcriptional levels [4]. Small noncoding RNAs (sRNAs), including small interfering RNA (siRNA), microRNA (miRNA) and Piwi-interacting RNA (piRNA), interact with the RNA-induced silencing complex (RISC) to regulate target gene expression at the post-transcriptional level [4-6]. Notably, microRNAs (miRNAs), which comprise one of the most abundant

\footnotetext{
* Correspondence: guangyou1963@yahoo.com.cn

1 Department of Parasitology, College of Veterinary Medicine,

Sichuan Agricultural University, Ya'an 625014, China

Full list of author information is available at the end of the article
}

classes of genes regulators [7], play important regulatory roles in metazoan animals and plants [8] by binding to complementary sequence in the 3 untranslated region (3-UTR) of target mRNAs, leading to cleavage or translational repression [2,9]. Mature miRNAs, derived from long primary stem-loop miRNA precursors approximately $70 \mathrm{bp}$ in length [10], regulate many processes, including apoptosis, cell proliferation, fat metabolism, hematopoiesis and organogenesis [11]. Since lin-4 was originally discovered in Caenorhabditis elegans [12,13], many miRNAs have been identified in almost all metazoan genomes, including flies, worms, plants, and mammals [14]. Furthermore, miRNAs are associated with animal development, including germline, muscle, and neuronal development $[15,16]$.

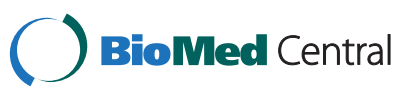

(c) 2013 Wu et al.; licensee BioMed Central Ltd. This is an Open Access article distributed under the terms of the Creative Commons Attribution License (http://creativecommons.org/licenses/by/2.0), which permits unrestricted use, distribution, and reproduction in any medium, provided the original work is properly cited. 
Taenia multiceps is a widespread cestode, which has two life cycle stages. The larval stage (coenurus) parasitizes the brain or spinal cord of domestic ruminants, such as buffalo, cattle, goats, horses, sheep, and yak, as well as wild species, causing lethal neurological symptoms [17]. Since human coenurosis was first reported by Brumpt in 1913 [18], further evidence has shown that this parasite causes zoonotic infections in humans [19-25]. Adult T. multiceps are found in the small intestine of dogs and other canids, and gravid proglottids in host feces are a source of infection following ingestion by an intermediate hosts [23].

Coenurus caused by $T$. multiceps occurs almost all over the world [26], especially in the developing countries of Africa and southeastern Asia, and causes huge economic losses from condemned meat and viscera [27]. Given the important regulatory functions of sRNAs in adapting to the environment and the lack of large-scale sRNA characterization in cestodes, it is important to investigate $T$. multiceps miRNAs and other sRNAs using high-throughput sequencing methods. In this study, we investigated $T$. multiceps miRNAs using an Echinococcus multilocularis reference genome and an adult T. multiceps transcriptome dataset. Our results increase the current understanding of the molecular mechanisms of cestode gene regulation [28], and will help to identify novel biomarkers by revealing the target genes [29,30] and develop new strategies to control parasitic zoonoses [31].

\section{Methods}

\section{Parasite preparation}

Larvae (coenuri) were collected from the brain of a naturally infected goat at an organic farm in Panzhihua, Sichuan, China. Infection was performed after morphological identification of the larvae. Two parasite-free beagles were orally infected with 20 larvae each. Forty-eight days after infection, adult T. multiceps were obtained from the small intestine of infected dogs after gravid proglottids were observed in their feces. T. multiceps were washed thoroughly in physiological saline solution $\left(37^{\circ} \mathrm{C}\right)$ to avoid host cell contamination, transferred into liquid nitrogen, and stored at $-80^{\circ} \mathrm{C}$.

All animals were handled in accordance with the Animal Protection Law of the People's Republic of China (a draft of which was released on September 18, 2009). This study was approved by the National Institute of Animal Health Animal Care and Use Committee at Sichuan Agricultural University (approval number 2010-018).

\section{Small RNA library preparation and Illumina sequencing}

Total RNA was extracted from adult $T$. multiceps $(n=6)$ using TRIZOL (Invitrogen, Carlsbad, CA), according to the manufacturer's protocol. RNA integrity was checked by determining the RNA integrity number using an Agilent 2100 Bioanalyzer. RNA sequences ranging from $18-30 \mathrm{nt}$ in length were isolated and purified from total RNA by Novex 15\% TBE-Urea gel (Invitrogen) electrophoresis. Proprietary (Solexa) adaptors were then added to the 3 and 5 -termini of sRNAs, which were then used for cDNA synthesis. These ligation products were amplified by reverse transcription PCR (RT-PCR) using a RTPCR kit (Invitrogen). PCR amplification products were purified for high-throughput sequencing by electrophoresis using a 6\% TBE PAGE gel (Invitrogen). The produced libraries were sequenced using a Solexa sequencer at the Beijing Genomics Institute (BGI)-Shenzhen, Shenzhen, China, according to the manufacturer's instruction.

\section{Bioinformatics analysis pipeline for searching conserved miRNAs}

The workflow for obtaining 'clean' reads involved filtering low quality tags; removing raw reads with 5' primer contaminants; trimming 3' adaptors; removing reads without insert tags; discarding reads with polyA tails; and removing contaminants formed by adaptor-adaptor ligation. The length distribution of clean reads was summarized, all clean reads were mapped to the E. multilocularis genome, which was obtained from the Sanger Institute FTP site [32], using the Short Oligonucleotide Alignment Package (SOAP) [33], and the expression and distribution of sRNAs in the genome were analyzed. Subsequently, clean reads were annotated against the Rfam database (version 10.0) [34] and GenBank noncoding RNA database [35] to remove noncoding RNAs, such as rRNA, scRNA, snoRNA, snRNA and tRNA. Tag2repeat software (provided by BGI) was used to select repeat overlapping sequences as repeat-associated small RNAs and then eliminate them. The remaining sequences were used to identify conserved miRNAs and predict novel miRNAs.

To identify conserved miRNAs in $T$. multiceps, clean small RNA sequences were aligned with the miRNA precursor and mature sequences of all animals deposited in miRBase 17.0, using tag2miRNA software (provided by BGI). Sequences containing no more than two mismatches with known miRNAs were defined as conserved miRNAs, and the most abundantly expressed sequence was selected when multiple sequences were assigned as the same conserved miRNA. Sequences that could not be annotated from sequence alignments were defined as non-annotation unique reads.

\section{Prediction of novel miRNAs}

The E. multilocularis genome [32] was also used to discover the potential miRNA precursors. Unannotated unique $T$. multiceps reads that perfectly matched the $E$. multilocularis genome were designated candidate miRNA precursors. Potential precursor sequences were 
selected from each end of the sequence matching the referenced genome, and MIREAP software [36] was used to determine whether these sequences form a characteristic miRNA hairpin-like structure, contain a Dicer cleavage site, and have a minimum free energy (MFE) lower than $18 \mathrm{kcal} / \mathrm{mol}$. miRNAs with these characteristics were defined as qualified precursors for candidate miRNAs. Previous studies suggest that miRNA precursors with a minimum free energy index (MFEI) greater than 0.85 are likely to be miRNAs $[37,38]$. We used the formula,

$$
\begin{aligned}
\text { MFEI }= & {[(\text { MFE } / \text { length of the RNA sequence }) \times 100] } \\
& \div(\mathrm{G}+\mathrm{C}) \%
\end{aligned}
$$

to select novel miRNAs with MFEIs $>0.85$.

\section{Reliability of conserved microRNAs and Validation of novel miRNA precursors}

Conserved miRNAs and novel candidate miRNA precursors were verified using a PCR-based method. Genomic DNA was extracted from adult $T$. multiceps using a Gentra Puregene Tissue Kit (Qiagen, Valencia, CA, USA) following the manufacture's protocol. Three conserved putative miRNA/miRNA* duplexes were selected randomly to check if their corresponding precursors form a characteristic pre-miRNA hairpin-like structure with low free energy and the putative miRNA in the stem. Using the candidate precursors which predicted based on the E. multilocularis genome, we designed primers for novel miRNA precursors and miRNA/ miRNA* duplexes using Primer Premier 5.0. Primers successfully used for amplifying the three conserved and 20 novel miRNA precursors are shown in Additional file 1. PCR was carried out according to the PCR verification scheme for pinewood nematodes [28]. Amplification product length was examined by $3.5 \%$ agarose gels electrophoresis using a 50 bp DNA ladder. Fragments between 60 and $100 \mathrm{nt}$ in length were subcloned into the pMD18-T vector (Takara, Dalian, Liaoning, China) for sequencing. RNAfold package [39] was used to predict the secondary structure of the amplified miRNA precursor.

\section{Prediction of miRNA target genes}

T. multiceps transcriptome unigenes obtained by Illumina sequencing were used to predict novel miRNA targets (Unigene: JR916739-JR948020 in the Transcriptome Shotgun Assembly Sequence Database at NCBI from our previous study [40]). miRNA target genes for adult $T$. multiceps were predicted using RNAhybrid $[41,42]$ parameters of $-\mathrm{f} 2,8-\mathrm{v} 3-\mathrm{u} 3$ (helix constraint, $2-8$ and maximal internal or bulge loop size per side, 3).
To understand the main biological functions and identify the biochemical metabolic pathway/signal transduction pathways of novel miRNAs target gene candidates, all target gene candidates were annotated against the Gene Ontology (GO) database [43] and the Kyoto Encyclopedia of Genes and Genomes (KEGG) database [44], as described in our recent research [45].

\section{Results}

\section{Illumina sequencing of small RNAs from T. multiceps}

We employed Illumina sequencing technology on a library of small RNAs from adult T. multiceps to identify miRNAs, which yielded 13.5 million raw reads. The dataset was deposited in NCBI Gene Expression Omnibus [46] with the accession number [GEO: GSE35647]. We removed 312,411 low quality tags, 68,068 3 adapter null reads, 111,184 reads without insert tags, 30,727 reads with 5 adapter contaminants, and 197,872 reads smaller than $18 \mathrm{nt}$, and 462 reads with ployA tails to obtain 12.8 million clean reads $(96.91 \%$ of the raw reads). The length distribution profile showed that small RNAs of 20-22 nt were the most abundant (Figure 1). After mapping all clean reads to the $E$. multilocularis genome (Additional file 2), 2,266,539 sRNAs (17.68\% of 12.8 million) were perfectly matched sequences in the E. multilocularis genome, including 39,765 unique sRNAs (2.88\% of total 1,379,196 unique sRNAs).

\section{Identification of microRNAs by computational algorithms} Non-coding small RNAs including 653,754 rRNAs, 1,086 snoRNAs, 12,806 snRNAs, and 728,898 tRNAs were removed after mapping all clean reads against the Rfam database (version 10.0). Subsequently, 392,874 total sRNAs (including 22,734 unique sRNAs) were identified as repeat associated sRNAs and then removed. A total of 11.2 million small RNA sequences were obtained. The distribution of total and unique sRNAs is shown in Figure 2. A total of 1,006 miRNAs (grouped into 796 miRNA families) were designated conserved miRNAs against miRBase 17.0. The expression levels and sequences of conserved miRNA families and miRNAs are shown in Additional file 3. Of these, 10 conserved miRNA families were identified in more than 100,000 counts: the five most abundant miRNA families were miR-71 (998,673 counts), miR-315 (774,438), miR-2840 (496,050), miR-1 (469,997), and miR-1422 $(254,527)$ (Table 1). Among the conserved miRNAs, we also identified 95 miRNAs* (antisense strands of miRNAs) (Additional file 3). Of these, $38 \mathrm{miRNAs}^{*}$ had $<10$ counts. The five most abundant miRNAs" were miR-1422f* $(254,460)$, miR-959* $(226,286)$, miR-133a* $(97,989)$, bantam* $(67,417)$, and miR-20a* $(16,529)$. Interestingly, for more than half of these miRNAs*, no complementary sequences (such as miR-959* and miR-133a*) were detected. 


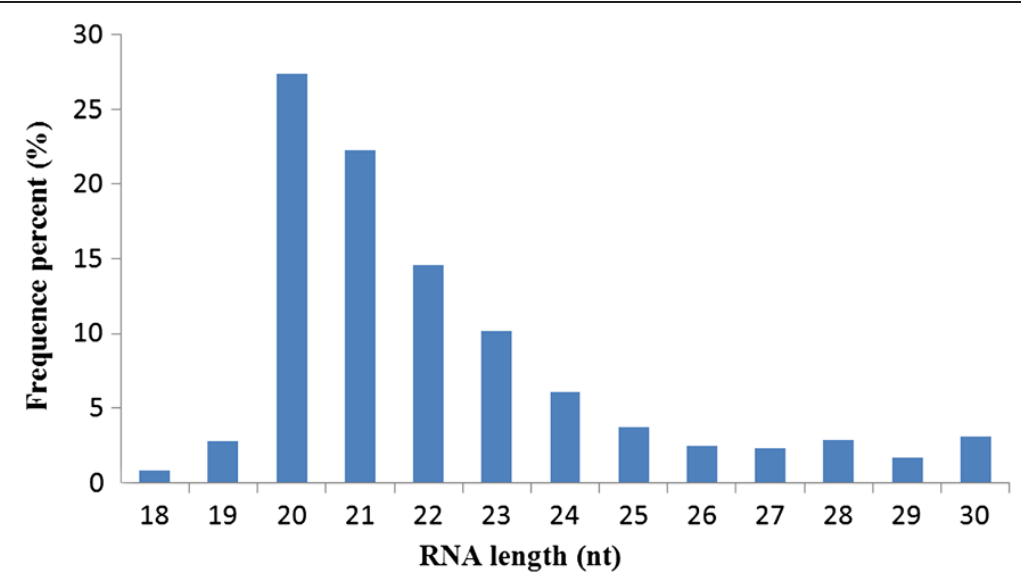

Figure 1 Length distribution analysis of sequenced small RNAs in Taenia multiceps. The frequencies of each unique read represent relative expression levels, and only small RNA sequences $18-30$ nt in length were considered.

This is inconsistent with a previous report that miRNAs* are less stable than mature miRNAs in locusts [4].

\section{Prediction of novel candidate miRNAs}

In addition to conserved miRNAs, we identified 20 candidate miRNAs. A total of 44 precursor candidates (comprising 36 different precursors, as two precursors mapped to multiple locations) were predicted using MIREAP software; these contained a Dicer cleavage site, and exhibited an appropriate secondary structure and MFE. Two candidate miRNAs mapped to multiple locations in the E. multilocularis genome: tmu-miR-0004 corresponds to eight different locations on different chromosomes, while tmu-miR-0039 corresponds to two different locations (Additional file 4A). In addition, tmumiR-0016 (designated tmu-novel-03) was the most abundant, with 9,463 reads, which suggests that it has an important role in adult $T$. multiceps. As a previous study on maize miRNAs suggested that miRNA precursors with
MFEIs $>0.85$ are most likely to be miRNAs [38], we determined MFEIs for all candidate miRNAs using the same formula. Following this analysis, 11 of the 36 candidates (MFEI $\geq 0.85$ ) were identified as confident novel miRNAs, including tmu-novel-01 (0.88), tmu-novel-03 (0.97), and tmu-novel-06 (0.98; others are shown in Additional file 4B).

\section{Experimental validation of miRNA precursors}

We used a PCR-based method to verify our predicted miRNA precursors, including three conserved miRNA/ miRNA* duplexes (tmu-miR-87/miR-87*; tmu-miR124b/miR-124b*; tmu-miR-2162/miR-2162*) and three novel miRNA/miRNA* duplexes (m0032, m0035, and m0041 were redesignated tmu-novel-14, novel-16, and novel-18, respectively). The electrophoretic analysis of three conserved miRNA precursor PCR products is shown in Figure $3 \mathrm{~A}$ and $3 \mathrm{~B}$. The hairpin structures of conserved miRNA precursors are displayed in Figure 3C,
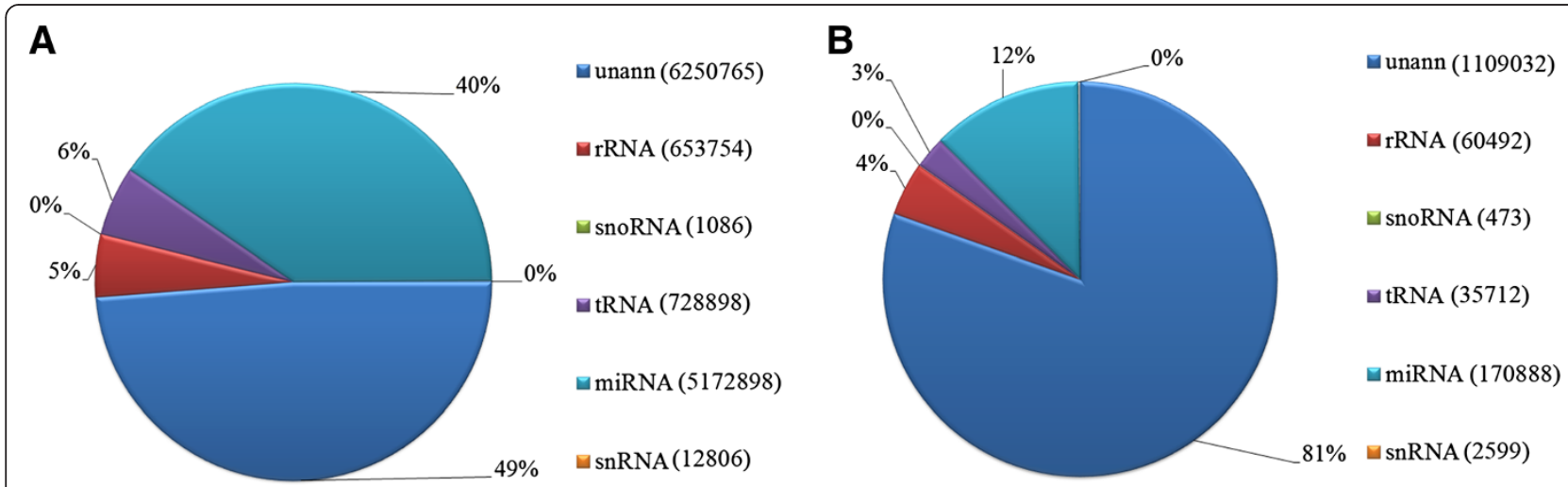

Figure 2 Distribution of small RNAs in Taenia multiceps annotation following Illumina sequencing. A. total reads. B. unique reads. "unann" stands for un-annotated reads/unique reads. One unique read means all the reads with the same sequence. 
Table 1 Sequences and abundance of the top 10 miRNA families in $T$. multiceps

\begin{tabular}{lll}
\hline miRNA family name & Sequence $^{\text {a }}$ & Counts \\
\hline miR-71 & TGAAAGACGATGGTAGTGAGA & 998673 \\
miR-315 & TTITGATTGTTGTGGGATGT & 774438 \\
miR-2840 & TAGGACTGGAAGACGGGAGA & 496050 \\
miR-1 & TGGAATGTTGTGAAGTATGT & 469997 \\
miR-1422 & AATAAAATAGTAGGACTGAT & 254527 \\
bantam & TGAGATCGCGATTACAGCTGAT & 227564 \\
miR-959 & TAAGGACTTGGGTGTAAAA & 226286 \\
miR-10 & CACCCTGTAGACCCGAGTTGA & 208265 \\
miR-4491 & AATTGTGGATTGATGGACCAAA & 176753 \\
miR-4175 & GGGATGTAGCTCAGTGGTAG & 150022 \\
\hline a miRNA sequence with the highest count in a single miRNA family. &
\end{tabular}

and the miRNA homologues in other species Echinococcus granulosus, E. multilocularis, Caenorhabditis elegans and Schistosoma japonicum from miRBase are listed in Figure 3D. In all, 20 out of 36 novel miRNA precursor candidates could be verified using gel electrophoresis (Figure 4). 19 of 20 verified miRNAs can be folded into characteristic miRNA stem-loop secondary hairpin structures (see Additional file 5). Of these, 15 sequenced T. multiceps miRNA precursors have $>90 \%$ similarity with the corresponding predicted sequences from $E$. multilocularis. However, one novel miRNA precursor (tmu-novel-19) has low similarity (65.1\%) and a different structure to the predicted E. multilocularis precursor, suggesting that this novel miRNA may have different functions in $T$. multiceps and $E$. multilocularis. Interestingly, six of the novel miRNAs did not share homology

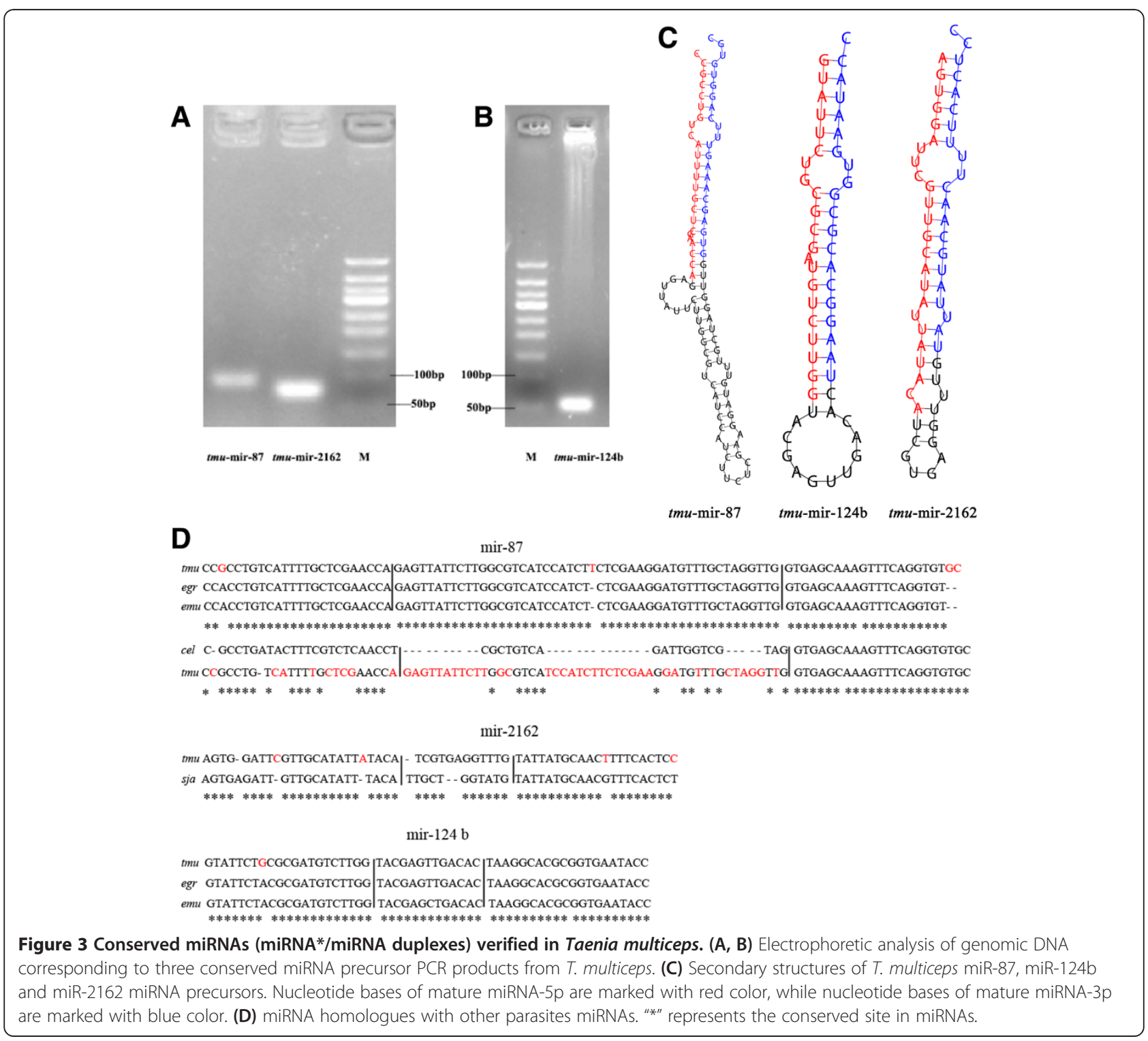




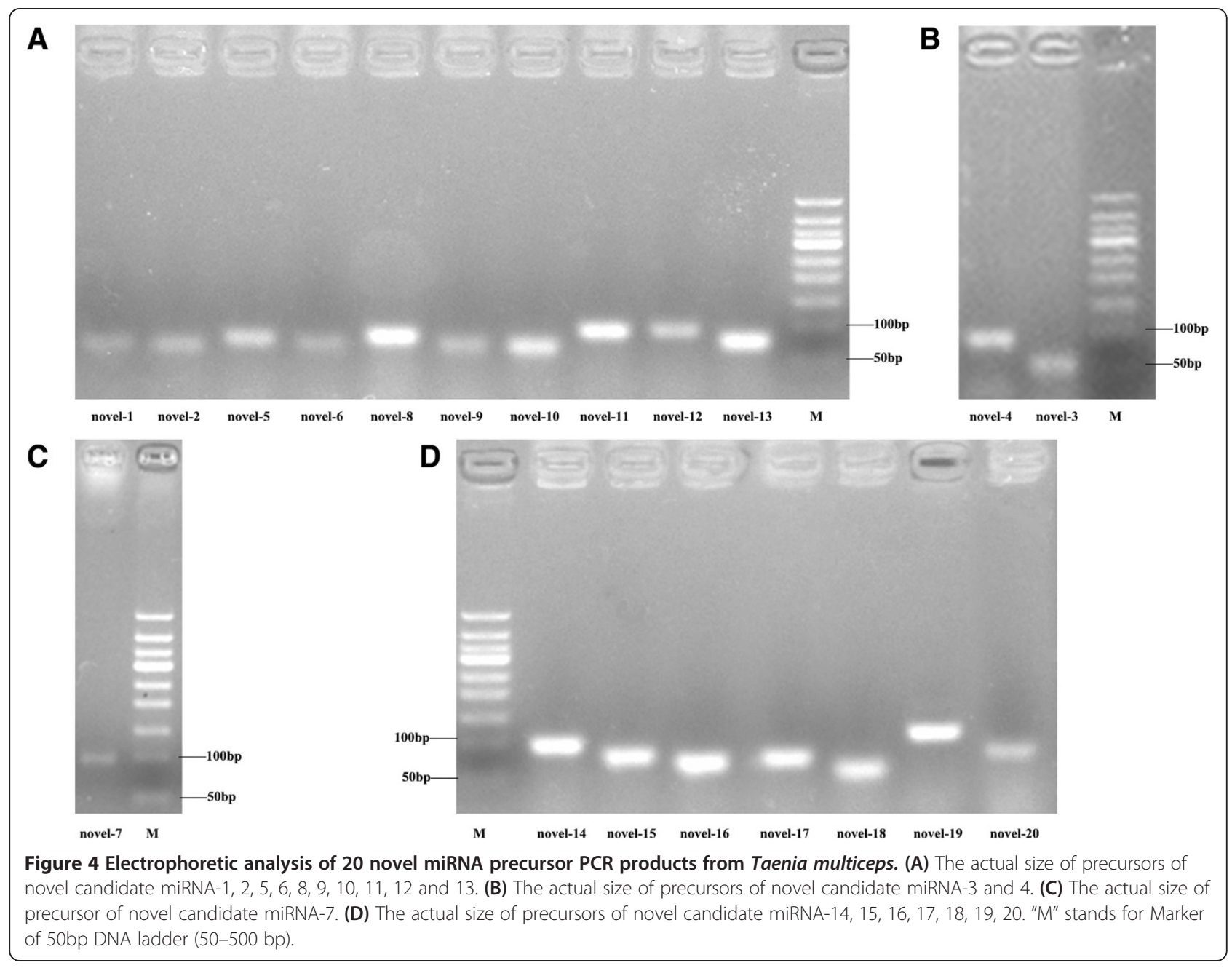

with sequences in the miRBase [47], and can therefore be considered specific miRNAs for Taeniidae (the sequences and structures of which are shown in Table 2 and Figure 5C), indicating they may play specific gene regulatory roles in Taeniidae cestodes. Additional file $4 \mathrm{C}$ showed the result of RNAfold software for predicting the secondary structure of the validated 23 (including 20 novel and 3 conserved) miRNA precursors.

\section{Target gene prediction}

To determine the functions of the 1,006 conserved miRNAs and 20 novel miRNAs in T. multiceps, we predicted their putative targets using a panel of 31,282 T. multiceps transcriptome unigenes; $10,121,914$ and 181,077 target unigene sites (Additional file 6), respectively, were obtained. Unigene 1299, annotated as a fatty acid binding protein (FABP), was targeted by tmu-novel-15. In addition, unigene 18109 , obtained by annotation of heat shock proteins (HSP), was targeted by tmu-novel-07. After mapping to the GO database, all putative target genes were classified into diverse GO functional groups. The GO functions of the predicted targets of novel miRNAs are shown in Additional file 7, Additional file 8, Additional file 9. In addition, KEGG pathway annotations revealed the biological functions of target unigenes of novel (Additional file 10) miRNAs.

\section{Discussion}

Animal miRNAs are important regulators of gene expression that function through imprecise complementarity to their mRNA targets [48]. Hence, it is essential to understand the functions of miRNAs throughout the lifecycle of parasites and determine how these may regulate host infection [49]. We identified adult T. multiceps miRNAs by Illumina sequencing. Since the adult worms used for RNA extraction were gravid, the identified miRNAs could also be expressed by the oncospheres. This dataset might help the further study of miRNAs of T. multiceps oncospheres. Our results showed that a low 


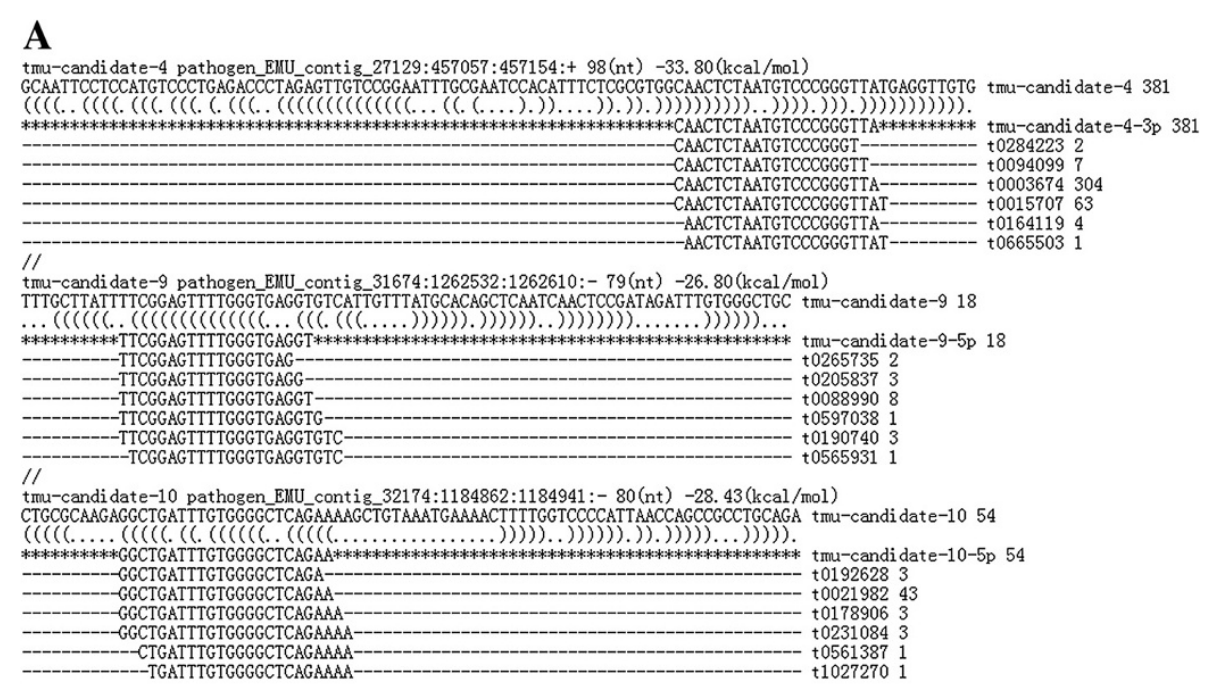

B tmu-candidate-12 pathogen_EMU_contig_32489:147847:147938:+ 92(nt) $-24.36(\mathrm{kcal} / \mathrm{mol})$
TTCTCTGTGTTAAATATATGTCTGCACTAGTAGTGTGTCTTCTTCTTCTTCAGGGAAGTCTCAGGACGCTACATTGGTAGCAGCGGAAAA tmu-candidate-12 7

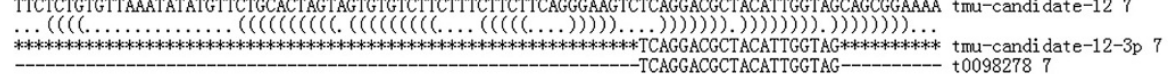
//
tmu-candidate-19 pathogen_EMU_contig_32811:493554:493624:- $71(\mathrm{nt})-20.60(\mathrm{kcal} / \mathrm{mol})$

tmu-candidate-19 pathogen_ElIU_contig_32811:493554:493624:- 71 (nt) $-20.60(\mathrm{kcal} / \mathrm{mol})$
ACGGCAGAGGAGTATTTGGGTGGAAGGTCACAAATGCAGTGATCACTGTGCCGGCGTATTTTAGTGTCAG tmu-candidate-19 141

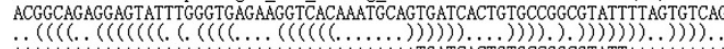

************************************************TGATCACTGTGCCGCGTATT************ tmu-candi date $-3 p 140$

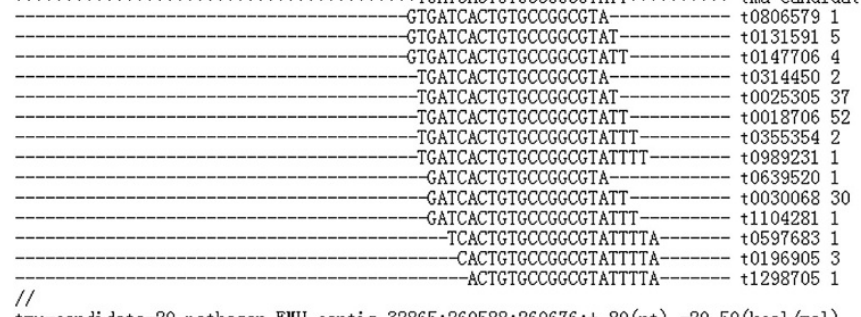

tmu-candidate-20 pathogen_EMU_contig_32865:260588:260676:+ 89(nt) $-20.50(\mathrm{kcal} / \mathrm{mol})$

CCGGGTTCCATTTAGCTAGTAGAGĀ̈GTGGAAGGGTGTTGCGTTTTCATATATTTGATATCTCCATGCAGGAACAGTGGACAGCTCCGT tmu-candidate-20 30

. $(((((((((((\ldots .((.(((((((.((.(((. .(((\ldots \ldots \ldots))) \ldots)))))))).) \ldots)))).) \ldots)))))) \ldots))))$.$) .$

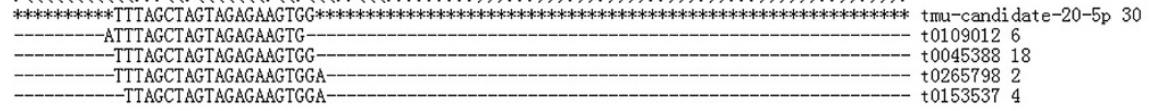
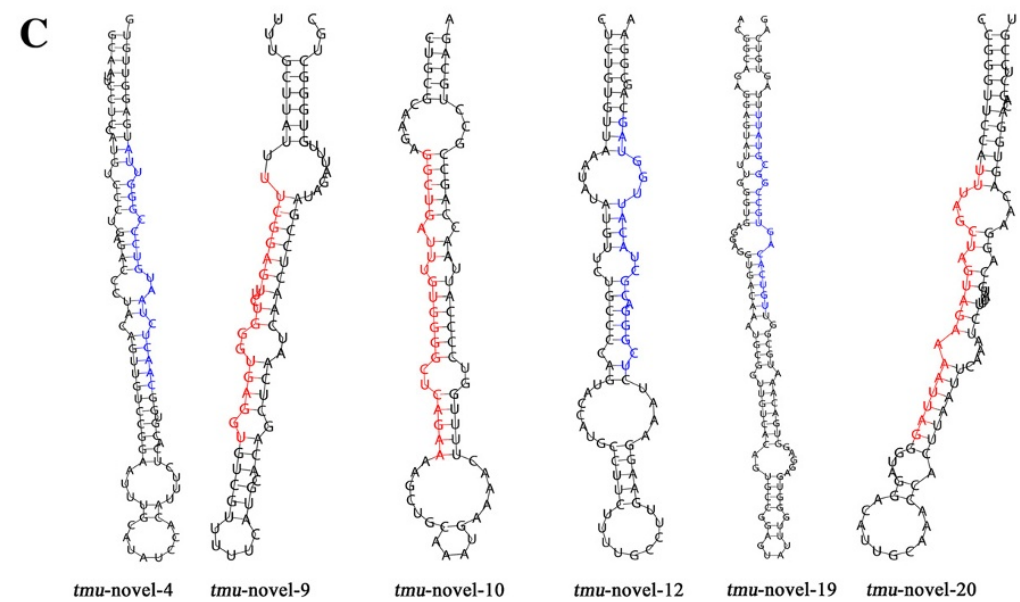

Figure 5 Predicted and validated precursors of six specific miRNA. (A,B) Location of putative miRNAs precursors in E. multilocularis genome. (C) Hairpin secondary structures of validated precursors of six species-specific miRNAs. Nucleotide bases of mature miRNA-5p (miRNA-3p) are marked with red (blue) color. Secondary structures were predicted by RNAfold package. 


\section{Table 2 Six validated specific miRNAs}

\begin{tabular}{|c|c|c|c|}
\hline $\begin{array}{l}\text { Candidate } \\
\text { name }\end{array}$ & Sequence & Count & Validated precursor \\
\hline tmu-novel-4 & CAACTCTAATGTCCCGGGTTA & 381 & GCAATTCCTCCATGTCCCTGAGACCCTACAGTTGTCCGGAATTTGCATATCCACATTTCTCACGTGGCAACTCTAATGTCCCGGGTTATGAGGTTGTG \\
\hline tmu-novel-9 & TTCGGAGTITGGGTGAGGT & 18 & TाTGCTTATTTCGGAGTTCTGGGTGAGGTGTCGTITTCATGCACAGCTCAATCAACTCCGATAGATTTGTGGGCTGC \\
\hline tmu-novel-10 & GGCTGATTTGTGGGGCTCAGAA & 54 & CTGCGCAAGAGGCTGATTTGTGGGGCTCAGAAAAGCTGCAAATGAAAACTTTGGTCCCCATTAACCAGCCGCCTGCAGA \\
\hline tmu-novel-12 & TCAGGACGCTACATTGGTAG & 7 & тTCTCTGTGTTAAATATATGTTCTGCCCCAGTACCATGCCTTCTTTTGCCTTGAAGGAAATCTCGGGACGCTACATTGGTAGCAGCGGAAAA \\
\hline tmu-novel-19 & TGATCACTGTGCCGGCGTATT & 140 & $\begin{array}{l}\text { ACGGCAGAGGAGTATTTGGGTGAGGAGGTGACAAATGCGGTTGTCACAGTGCCGGAGTATTTGGGTGAGGAGGTGACAAATGCGGTTGTCACAGTGCCG } \\
\text { GCGTATTTAGTGTCAG }\end{array}$ \\
\hline tmu-novel-20 & TTIAGCTAGTAGAGAAGTGG & 30 & CCGGGTTCCATTTAGCTAGTAGAAAATTAGGGTAGGACATTGCAAACCACTTAATTCAAATCTTTATGCAGGAACAGTGGACAGCTCCGT \\
\hline
\end{tabular}

"Count" means the amount of the candidate novel miRNA. "Validated precursor" shows the corresponding precursor sequence obtained from genomic PCR validation. 

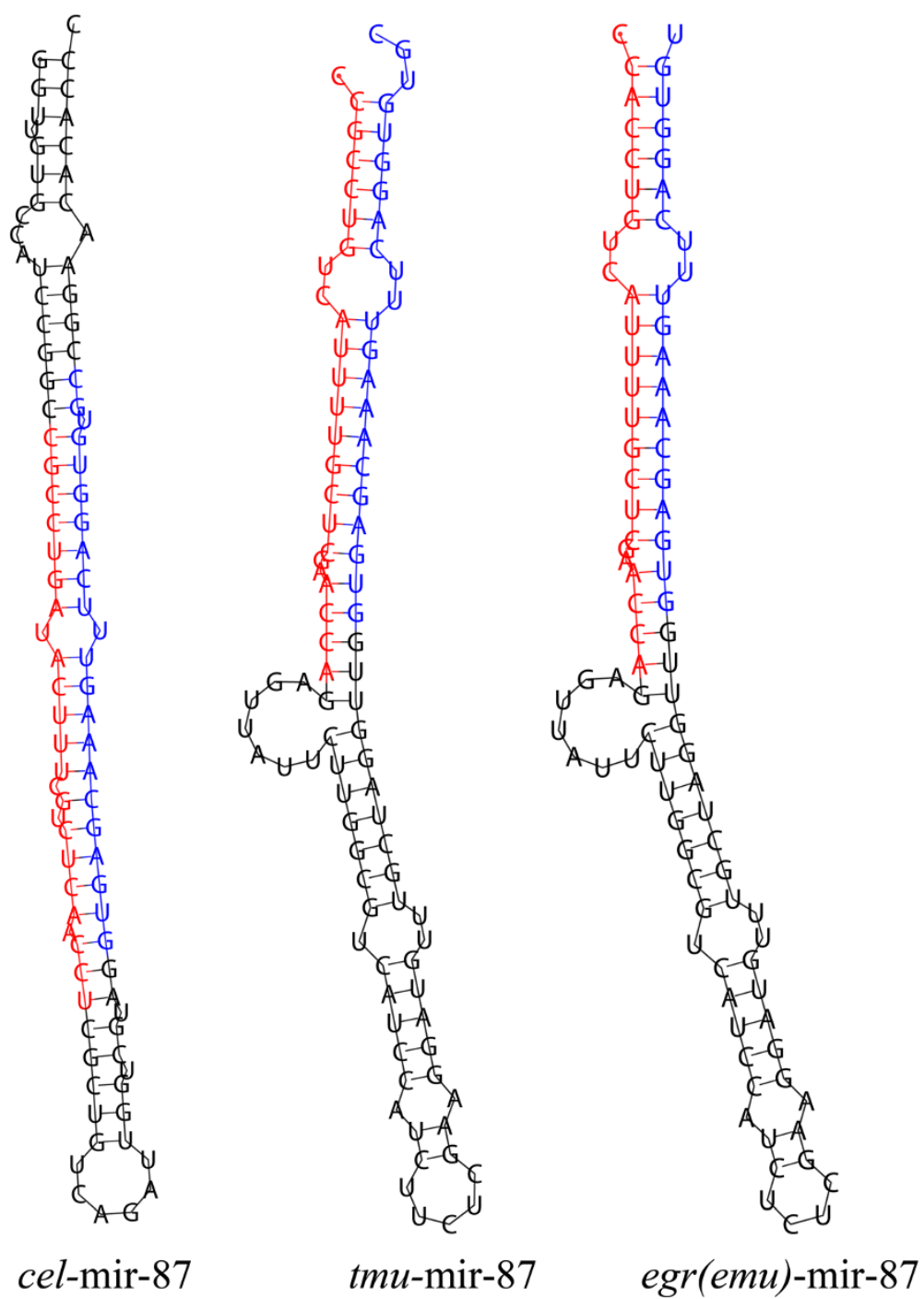

Figure 6 Comparison the secondary structures of miR-87 precursor in C. elegans, T. multiceps, E. granulosus and E. multilocularis. The tmu-mir-87 shares the similar secondary structure with egr-mir-87 and emu-mir-87, but differs from the cel-mir-87. Nucleotide bases of mature miRNA-5p are marked with red color, while nucleotide bases of mature miRNA-3p are marked with blue color.

percentage $(17.68 \%)$ of reads had a perfect match to sequences and only 39,765 out of a total of 1,379,196 (19.46\%) unique sRNAs matched sequences in the related E. multilocularis genome, as proposed in previous study of Clonorchis sinensis sRNAs when matching which into the related genome of Schistosoma japonicum [31]. This lack of homology may be due to using the E. multilocularis reference genome, as the T. multiceps whole-genome sequence is currently unavailable. $T$. multiceps and E. multilocularis are both Taeniidae cestodes, but significant differences exist between them [50].

Some kinds of miRNAs were expressed with high predominance. A previous data showed that known miRNAs of Taenia saginata were expression predominated by miR-71 (69.53\% of the total reads) [51], which was also detected to be the most abundant conserved miRNA in adult T. multiceps (16.43\%). Similar to T. saginata, members of the common miR-40 family were not detected in T. multiceps in this study. However, low expression of 12 members of another common miRNA family, let-7 (not found in T. saginata [51]), was shared among T. multiceps (17,125 counts), E. granulosus and E. multilocularis. Considering that $T$. saginata and $T$. multiceps belong to the same genus, Taenia, the absence of let-7 miRNAs in $T$. saginata, their presence in T. multiceps and their universal existence in some other species suggest that 
non-recovery of let-7 in T. saginata may be attributed to the specific stage of the parasite examined or experimental methods. In addition, we compared conserved miRNAs of T. multiceps with E. granulosus (E. multilocularis) which were obtained from miRBase. The miR-8, which was found in E. granulosus and E. multilocularis, was not identified in adult T. multiceps. While miR-96 was found both in E. multilocularis and T. multiceps but not in $E$. granulosus. Among these three species, two other miRNAs (miR-4990 and miR-4991) were only detected in E. granulosus. These findings could be useful for miRNAs' function researches.

The miR-1, a highly conserved muscle-specific miRNA, is represented in the top four abundant T. multiceps miRNAs. Target searching identified many unigene targets of miR-1, including unigenes 754 and 757 , both of which have been annotated as malate dehydrogenase. This finding is consistent with a recent report that malate dehydrogenase is regulated by miR-1 in Trichomonas vaginalis [52], illustrating that our method for miRNA target prediction was correcet. As malate dehydrogenase participates in the citric acid cycle [53] and T. multiceps are intestinal parasites that produce ATP mainly through anaerobic glycolysis (not the citric acid cycle) [54], it will be interesting to investigate how miR-1 and malate dehydrogenase interact in T. multiceps. However, we can infer that miR-1 may interact with other miRNAs to regulate glucose metabolism in T. multiceps.

To make sure the validity of the identified conserved miRNAs, we randomly selected three conserved miRNA/miRNA* duplexes for validation. However, the on-going precursor validation and detailed analyses of miRNAs' structure and function are required in further studies. Compared with the cel-miR-87 from miRBase, the mature miRNA tmu-miR-87-5p seemed not so highly conserved (Figure $3 \mathrm{D}$ ) and the secondary structure of tmu-miR-87 precursor seemed abnormal (Figure 6). However, compared with egr-miR-87 and emu-miR-87, the sequence of mature miRNA tmu-miR-87-5p was highly conserved with only one nucleotide variation (Figure 3D) and the hairpin structure was extremely similar (Figure 6).

As miRNAs* are less stable than mature miRNAs, being quickly degraded after mature miRNA enters the RISC complex $[4,9]$, we did not expect these molecules to be sequenced at a high frequency. However, more

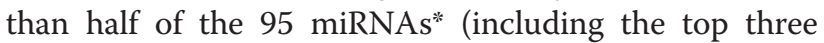
abundant miRNAs*: miR-1422f*, miR-959*, and miR133a*) were sequenced with high frequency in this study without their corresponding mature strands (miR-1422f, miR-959 and miR-133a) detected. Furthermore, many miRNAs* (e.g., miR-20a* and miR-216b*) were sequenced at similar frequencies to their corresponding mature miRNAs (miR-20a and miR-216b). This suggests that
miRNAs* may play functional roles in T. multiceps and that small RNA precursors may form two different regulatory small RNAs, as has been proposed by previous studies in E. granulosus and Drosophila melanogaster [6,55]. The miR-1422 family is the second largest family in C. sinensis, consisting of 13 members [31]. Although miR-1422f* was the most abundant miRNA* sequenced in T. multiceps, only two miR-1422 family members (miR$1422 \mathrm{f}^{*}$ and miR-1422j) were detected. The miR-1422 family reads therefore suggest a strong bias (99.97\%) toward miR-1422* in adult T. multiceps. miR-959*, whose mature strand has only previously been reported in D. melanogaster [56], was the second most abundant miRNA* in T. multiceps.

We identified 20 candidate novel T. multiceps miRNAs using PCR-based methods, thus providing evidence that computational methods used for miRNA prediction were correctly executed. We also verified that miRNA precursor and flanking sequences could be folded into typical miRNA-like hairpin structures. The sequences and structures of these newly candidate miRNAs should help future studies of miRNA function in T. multiceps. All 20 novel miRNAs are regarded as candidate novel miRNAs here. Additional evidence is required to further validation their reliability. However, we failed to validate 16 other predicted novel miRNAs, including two candidate miRNAs with multiple loci in the E. multilocularis genome. We consider that this may be due to 1 ). the poor quality of primers and the difficulty of primer designing as we designed primers to amplify full-length predicted precursors. 2). the miRNA precursors were predicted by comparing to a related cestode species E. multilocularis instead of $T$. multiceps, which may highlight differences between the cestode species at the nucleotide level.

More importantly, we discovered six specific miRNAs with different structures in T. multiceps compared to E. multilocularis genome. As they both belong to Taeniidae, we speculate that these specific miRNAs might have specialized functions related to cellular processes in Taeniidae, and may therefore provide novel therapeutics for this disease [49]. Our identification of three candidate novel miRNA/miRNA* pairs in T. multiceps (Additional file 4B), m0032, m0035, and m0041 (predicted by computational methods), equivalent to the PCRvalidated $t m u$-novel-14, tmu-novel-16 and tmu-novel-18, respectively, provides more supporting evidence that these are authentic miRNAs. Interestingly, the precursor of the 13 low-confidence candidate novel miRNA $(\mathrm{MFEI}<0.85)$ determined using the MFEI formula could be validated by PCR-based method and meeting the secondary structure and the required MFE (Additional file 2). This is likely to be due to the differences that exist between animal and plant miRNAs [57]. Together with our recent study of Dirofilaria immitis miRNAs identification [58], these 
results indicate that the MFEI formula for plant miRNAs may not be suitable for selecting high-confidence animal miRNAs, or at least not $T$. multiceps and $D$. immitis miRNAs.

It was a challenge to predict miRNAs targets in $T$. multiceps, as the whole-genome sequence of T. multiceps is currently unavailable and $T$. multiceps expressed sequence tags (ESTs) are lacking. In previous miRNA studies of locust and pinewood nematode, researchers chose EST databases to predict miRNA targets $[4,28]$. We used T. multiceps transcriptome unigenes obtained by highthroughput sequencing as an alternative method to predict miRNA targets because animal miRNAs can bind to a broad spectrum of different target mRNAs through imprecise base pairing [48]; and unigene populations are derived from mRNAs expressed under specific conditions [59]. A large number of the 181,077 target sites in 31,282 adult $T$. multiceps unigenes were predicted by only 20 candidate novel miRNAs. This indicates that a single novel miRNA may have a large number of unigene target sites, thus suggesting that miRNAs have diverse functions. For example, we identified 8,665 target sites in multiple unigenes for the most abundant novel miRNA, tmunovel-03. Most unigenes were predicted to contain more than one target site for different novel miRNAs. For example, unigene 17356, annotated as phosphoenolpyruvate carboxykinase (PEPCK), was targeted by both tmunovel-01 and tmu-novel-11 (Additional file 6). As we discussed in our previous study of transcriptome that the lack of this important enzyme would interrupt $T$. multiceps glycometabolism [53], we presume that tmu-novel-1 and tmu-novel-11 play important roles in regulating PEPCK executive functions in T. multiceps. We showed all the target genes we got in Additional file 6. Nevertheless, further validation of putative miRNA targets is required.

\section{Conclusions}

In this study, adult $T$. multiceps miRNAs were characterized and identified using Illumina sequencing to provide a platform for further research into the regulation of gene networks in this organism. Our discovery of novel miRNAs, including six specific miRNAs for Taeniidae, in adult $T$. multiceps may help to develop new therapeutic approaches for diseases caused by this parasite. We predicted target mRNAs for 20 candidate novel miRNAs and investigated the functions of target gene candidates in adult T. multiceps. Ongoing work is needed to verify the remaining 16 candidate novel miRNAs, validate their target genes, and elucidate the functions of newly identified T. multiceps miRNAs.

\section{Availability of supporting data}

The dataset of small RNAs (13.5 million raw reads) from adult $T$. multiceps was deposited in NCBI Gene
Expression Omnibus [46] with the accession number [GEO: GSE35647].

\section{Additional files}

\begin{abstract}
Additional file 1: PCR primers for three conserved and 20 novel Taenia multiceps miRNA precursors. A total of 23 pairs of primers were used successfully in validation experiments.

Additional file 2: Distribution of Taenia multiceps small RNAs across different chromosomes of Echinococcus multilocularis. "sense" and "anti-sense" represent "+" and "-" strands of Chromosomes, respectively.

Additional file 3: Conserved Taenia multiceps miRNAs based on miRBase 17.0. (A) Conserved miRNA families and miRNAs (B) List of Taenia multiceps antisense miRNAs (miRNAs*).
\end{abstract}

Additional file 4: Details of the novel miRNA precursors. (A) Novel miRNA precursors predicted by MIREAP software using the Echinococcus multilocularis genome. (B) Confidence level of novel miRNAs selected using the minimal folding free energy index. (C) The result of RNAfold software for predicting the secondary structure of validated miRNA precusors.

Additional file 5: Hairpin secondary structures of $\mathbf{2 0}$ novel Taenia multiceps miRNA precursors. Nucleotide bases of mature miRNA-5p (miRNA-3p) are marked with red (blue) color. The stem-loop structure of all validated novel miRNA precursors was predicted by RNAfold software. "Tmu-novel candidate-17" represents the non-typical secondary structure. Three novel miRNA/miRNA* pairs (tmu-novel-14, tmu-novel-16 amd tmunovel-18) were validated. All 20 novel miRNAs noted in this Figure are the candidate names of novel miRNAs of T. multiceps.

Additional file 6: Novel miRNA target sites conserved in Taenia multiceps unigenes. 181,077 target unigenes were predicted for 20 novel miRNAs based on 31,282 adult T. multiceps transcriptome, which were obtained by Illumina sequencing and Trinity assembling.

Additional file 7: Cellular component GO annotations for candidate target unigenes for novel Taenia multiceps miRNAs. 5,696 target unigenes were assigned to 324 GO-terms from "Cellular component" ontology. "Gene Ontology term" means GO terms from Component Ontology with P-value as good as or better than 1. "Cluster frequency" stands for number and frequency of target unigenes related to this term. "Genome frequency of use" represents number and frequency of coding genes related to this term.

Additional file 8: Molecular function GO annotations for candidate target unigenes of novel Taenia multiceps miRNAs. 7,095 target unigenes were assigned to 604 GO-terms from "Molecular function" ontology.

Additional file 9: Biological process GO annotations for candidate target unigenes of novel Taenia multiceps miRNAs. 6,178 target unigenes were assigned to 2,262 GO-terms from "Biological process" ontology.

Additional file 10: KEGG pathways for predicted target genes of Taenia multiceps novel miRNAs. 9,306 target unigenes were assigned into 240 KEGG pathways. . "Pvalue" and "Qvalue" represent P-value before correction and corrected $\mathrm{P}$-value, respectively.

\section{Competing interests}

The authors declare that they have no competing interests.

\section{Authors' contributions}

XHW and GYY conceived and designed the whole experiment. DYY, RHZ, WPZ, NW and JHW performed the experiments of verification. NY, HMN and YX analyzed the data. XBG, SXW and XRP contributed reagents/materials/ analysis tools. XHW and YF drafted the manuscript. All authors read and approved the final manuscript.

\section{Acknowledgements}

This work was supported by the Program for Changjiang Scholars and Innovative Research Team in University (PCSIRT) (No. IRT0848). We would like to thank Beijing Genomics Institute-Shenzhen, Shenzhen, People's Republic 
of China for their assistance with Illumina sequencing and related bioinformatics analyses. We also wish to extend our deep thank to Zhuol Guo for his technical assistance and useful comments on the figures.

\section{Author details}

'Department of Parasitology, College of Veterinary Medicine, Sichuan Agricultural University, Ya'an 625014, China. 'Department of Chemistry, College of Life and Basic Science, Sichuan Agricultural University, Ya'an 625014, China.

Received: 11 April 2013 Accepted: 8 August 2013

Published: 13 August 2013

\section{References}

1. Alvarez-Garcia I, Miska EA: MicroRNA functions in animal development and human disease. Development 2005, 132(21):4653.

2. Kato M, De Lencastre A, Pincus Z, Slack FJ: Dynamic expression of small non-coding RNAs, including novel microRNAs and piRNAs/21U-RNAs, during Caenorhabditis elegans development. Genome Biol 2009, 10(5):R54

3. Kloosterman WP, Plasterk RHA: The diverse functions of microRNAs in animal development and disease. Dev Cell 2006, 11(4):441-450.

4. Wei $Y$, Chen S, Yang $P, M a ~ Z$, Kang L: Characterization and comparative profiling of the small RNA transcriptomes in two phases of locust. Genome Biol 2009, 10(1):R6.

5. Fejes-Toth K, Sotirova V, Sachidanandam R, Assaf G, Hannon GJ, Kapranov P, Foissac S, Willingham AT, Duttagupta R, Dumais E: Post-transcriptional processing generates a diversity of $5^{\prime}$-modified long and short RNAs: Affymetrix/Cold Spring Harbor Laboratory ENCODE Transcriptome Project*. Nature 2009, 457(7232):1028.

6. Ghildiyal M, Zamore PD: Small silencing RNAs: an expanding universe. Nat Rev Genet 2009, 10(2):94-108

7. Berezikov E, Guryev V, van de Belt J, Wienholds E, Plasterk RH, Cuppen E: Phylogenetic shadowing and computational identification of human microRNA genes. Cell 2005, 120(1):21-24.

8. Filipowicz W, Jaskiewicz L, Kolb FA, Pillai RS: Post-transcriptional gene silencing by siRNAs and miRNAs. Curr Opin Struct Biol 2005, 15(3):331-341.

9. Bartel DP: MicroRNAs: genomics, biogenesis, mechanism, and function. Cell 2004, 116(2):281-297.

10. Lee $Y$, Jeon K, Lee JT, Kim S, Kim VN: MicroRNA maturation: stepwise processing and subcellular localization. EMBO J 2002, 21(17):4663-4670.

11. Nelson P, Kiriakidou M, Sharma A, Maniataki E, Mourelatos Z: The microRNA world: small is mighty. Trends Biochem Sci 2003, 28(10):534-540.

12. Lee RC, Feinbaum RL, Ambros V: The C. elegans heterochronic gene lin- 4 encodes small RNAs with antisense complementarity to lin-14. Cell 1993, 75(5):843-854

13. Wightman $\mathrm{B}, \mathrm{Ha}$ I, Ruvkun $\mathrm{G}$ : Posttranscriptional regulation of the heterochronic gene lin-14 by lin- 4 mediates temporal pattern formation in C. elegans. Cell 1993, 75(5):855-862.

14. He L, Hannon GJ: MicroRNAs: small RNAs with a big role in gene regulation. Nat Rev Genet 2004, 5(7):522-531.

15. Carmi I: Molecular Biology Select. Cell 2006, 126(2):223-225.

16. Stefani G, Slack FJ: Small non-coding RNAs in animal development. Nat Rev Mol Cell Biol 2008, 9(3):219-230.

17. Edwards G, Herbert l: Observations on the course of Taenia multiceps infections in sheep: clinical signs and post-mortem findings. Br Vet J 1982, 138(6):489.

18. E. B: Precis de parasitologie. 2nd edition. Paris: Masson and Co; 1913:281.

19. Becker B, Jacobson S: Infestation of the human brain with Coenurus cerebralis; a report of three cases. Lancet 1951, 2(6675):198.

20. Benger A, Rennie R, Roberts J, Thornley J, Scholten T: A human coenurus infection in Canada. Am J Trop Med Hyg 1981, 30(3):638.

21. Crusz H: On an English case of an intramedullary spinal Coenurus in Man, with some remarks on the identity of Coenurus spp. infesting Man. J Helminthol 1948, 22(2):73-78.

22. Hermos JA, Healy GR, Schultz MG, Barlow J, Church WG: Fatal human cerebral coenurosis. JAMA 1970, 213(9):1461

23. Ing MB, Schantz PM, Turner JA: Human coenurosis in North America: case reports and review. Clin Infect Dis 1998, 27(3):519-523.

24. Wainwright J: Coenurus cerebralis and racemose cysts of the brain. J Pathol Bacteriol 1957, 73(2):347-354

25. Wilson V, Wayte D, Addae R: Human coenurosis-The first reported case from Ghana. Trans R Soc Trop Med Hyg 1972, 66(4):611-623.
26. Gauci C, Vural G, Oncel T, Varcasia A, Damian V, Kyngdon CT, Craig PS, Anderson GA, Lightowlers MW: Vaccination with recombinant oncosphere antigens reduces the susceptibility of sheep to infection with Taenia multiceps. Int J Parasitol 2008, 38(8-9):1041-1050.

27. Sharma D, Chauhan P: Coenurosis status in Afro-Asian region: a review. Small Rumin Res 2006, 64(3):197-202.

28. Huang QX, Cheng XY, Mao ZC, Wang YS, Zhao LL, Yan X, Ferris VR, Xu RM, Xie BY: MicroRNA discovery and analysis of pinewood nematode Bursaphelenchus xylophilus by deep sequencing. PLoS One 2010, 5(10):e13271

29. Gilad S, Meiri E, Yogev Y, Benjamin S, Lebanony D, Yerushalmi N, Benjamin $\mathrm{H}$, Kushnir M, Cholakh H, Melamed N: Serum microRNAs are promising novel biomarkers. PLoS One 2008, 3(9):e3148.

30. Mitchell PS, Parkin RK, Kroh EM, Fritz BR, Wyman SK, Pogosova-Agadjanyan EL, Peterson A, Noteboom J, O'Briant KC, Allen A: Circulating microRNAs as stable blood-based markers for cancer detection. Proc Nat Sci Acad 2008, 105(30):10513

31. Xu MJ, Liu Q, Nisbet A, Cai XQ, Yan C, Lin RQ, Yuan ZG, Song HQ, He XH, Zhu XQ: Identification and characterization of microRNAs in Clonorchis sinensis of human health significance. BMC genomics 2010, 11(1):521.

32. The Sanger Institute FTP site. [http://www.sanger.ac.uk/resources/downloads/ helminths/echinococcus-multilocularis.html]

33. Li R, Li Y, Kristiansen K, Wang J: SOAP: short oligonucleotide alignment program. Bioinformatics 2008, 24(5):713-714

34. Rfam database. [http://www.sanger.ac.uk/software/Rfam]

35. The GenBank noncoding RNA database. [http://www.ncbi.nlm.nih.gov/]

36. The MIREAP software. [http://sourceforge.net/projects/mireap/]

37. Zhang B, Pan X, Cox S, Cobb G, Anderson T: Evidence that miRNAs are different from other RNAs. Cell Mol Life Sci 2006, 63(2):246-254.

38. Wang L, Liu H, Li D, Chen H: Identification and characterization of maize microRNAs involved in the very early stage of seed germination. BMC genomics 2011, 12(1):154.

39. Hofacker IL: Vienna RNA secondary structure server. Nucleic Acids Res 2003, 31(13):3429-3431

40. Wu X, Fu Y, Yang D, Zhang $R$, Zheng W, Nie H, Xie Y, Yan N, Hao G, Gu X, Wang S, Peng X, Yang G: Detailed Transcriptome Description of the Neglected Cestode Taenia multiceps. PLoS One 2012, 7(9):e45830.

41. Rehmsmeier M: Prediction of microRNA targets. Methods Mol Biol 2006 342:87.

42. Rehmsmeier M, Steffen $P$, Höchsmann M, Giegerich R: Fast and effective prediction of microRNA/target duplexes. RNA 2004, 10(10):1507-1517.

43. The Gene Ontology. http://www.geneontology.org/.

44. The Kyoto encyclopedia of genes and genomes database. http://www. genome.jp/kegg

45. Fu Y, Lan J, Zhang Z, Hou R, Wu X, Yang D, Zhang R, Zheng W, Nie H, Xie Y, Yan N, Yang Z, Wang C, Luo L, Liu L, Gu X, Wang S, Peng X, Yang G: Novel Insights into the Transcriptome of Dirofilaria immitis. PLoS One 2012, 7(7):e41639.

46. GEO of NCBI. [http://www.ncbi.n/m.nih.gov/geo/]

47. The microRNA database. [http://www.mirbase.org/]

48. Ambros V: The functions of animal microRNAs. Nature 2004 431(7006):350-355.

49. Liu Q, Tuo W, Gao H, Zhu XQ: MicroRNAs of parasites: current status and future perspectives. Parasitol Res 2010, 107(3):501-507.

50. De Wit E, Linsen SEV, Cuppen E, Berezikov E: Repertoire and evolution of miRNA genes in four divergent nematode species. Genome Res 2009 19(11):2064-2074.

51. Ai L, Xu M, Chen M, Zhang Y, Chen S, Guo J, Cai Y, Zhou X, Zhu X, Chen J: Characterization of microRNAs in Taenia saginata of zoonotic significance by Solexa deep sequencing and bioinformatics analysis. Parasitol Res 2011, 110:2373-2378.

52. Lin WC, Huang KY, Chen SC, Huang TY, Chen SJ, Huang PJ, Tang P: Malate dehydrogenase is negatively regulated by miR-1 in Trichomonas vaginalis. Parasitol Res 2009, 105(6):1683-1689.

53. Li G: Biochemistry of parasites: energy metabolism. In Advanced parasitology. Volume 1. 1st edition. Edited by Li G, Xie M. China: High Education Press; 2007:109.

54. Pan Q: Trypanosome: host interactions. In Molecular parasitology. Volume 1. 1st edition. Edited by Pan Q, Tang L. China: Shanghai Science and Technology Press; 2004:353.

55. Cucher M, Prada L, Mourglia-Ettlin G, Dematteis S, Camicia F, Asurmendi S, Rosenzvit M: Identification of Echinococcus granulosus microRNAs and 
their expression in different life cycle stages and parasite genotypes. Int J Parasitol 2011, 41(3-4):439-448.

56. Stark A, Kheradpour P, Parts L, Brennecke J, Hodges E, Hannon GJ, Kellis M: Systematic discovery and characterization of fly microRNAs using 12 Drosophila genomes. Genome Res 2007, 17(12):1865-1879.

57. Millar AA, Waterhouse PM: Plant and animal microRNAs: similarities and differences. Funct Integr Genomics 2005, 5(3):129-135.

58. Fu Y, Lan J, Wu X, Yang D, Zhang Z, Nie H, Hou R, Zhang R, Zheng W, Xie Y, Yan N, Yang Z, Wang C, Luo L, Liu L, Gu X, Wang S, Peng X, Yang G: Identification of Dirofilaria immitis miRNA using illumina deep sequencing. Veterinary Res 2013, 44:3.

59. Costa V, Angelini C, De Feis I, Ciccodicola A: Uncovering the complexity of transcriptomes with RNA-Seq. J Biomed Biotechnol 2010, 2010:853916.

doi:10.1186/1746-6148-9-162

Cite this article as: Wu et al:: Identification of neglected cestode Taenia multiceps microRNAs by illumina sequencing and bioinformatic analysis. BMC Veterinary Research 2013 9:162.

\section{Submit your next manuscript to BioMed Central and take full advantage of:}

- Convenient online submission

- Thorough peer review

- No space constraints or color figure charges

- Immediate publication on acceptance

- Inclusion in PubMed, CAS, Scopus and Google Scholar

- Research which is freely available for redistribution 